\title{
Autocompaixão, bem-estar subjetivo e estado de saúde na idade avançada
}

\author{
Self-compassion, well-being, and health in advanced age
}

Artigo Original | Original Article

\author{
Liliana Parente, PsyM (1a), Marina Cunha, PhD (1,2b), Ana Galhardo, PhD (1,2c), Margarida Couto, PhD (1c) \\ (1) Instituto Superior Miguel Torga, Coimbra, Portugal \\ (2) Centro de Investigação do Núcleo de Estudos e Intervenção Cognitivo-Comportamental da Faculdade de Psicologia e de Ciências da Educação da Universidade de \\ Coimbra, Portugal \\ (a) Elaboração do trabalho, recolha e inserção de dados, análise estatística. \\ (b) Coordenação do projeto, elaboração do trabalho, análise estatística e revisão do texto. \\ (c) Elaboração e revisão do texto.
}

Autor para correspondência | Corresponding author: Liliana Parente; liliana.fd.parente@gmail.com

\begin{abstract}
RESUMO
Palavras-Chave

Autocompaixão

Saúde física

Saúde mental

Bem-estar subjetivo

Satisfação com a vida

Introdução: $\mathrm{O}$ aumento da população envelhecida constitui um avanço na sociedade, mas também um grande desafio, impondo a necessidade de ações que promovam um envelhecimento bem-sucedido.

Objetivos: Analisar de que forma é que a autocompaixão, satisfação com a vida, afetos, estado de saúde físico e mental se encontram associados na idade avançada, controlando ainda o efeito do sexo, idade, escolaridade, local de residência e tipo de resposta social dos participantes. Explorar qual o conjunto de variáveis que melhor prediz a satisfação com a vida e o estado de saúde nos idosos.

Método: 155 indivíduos, com idades entre os 65 e 94 anos, dos distritos de Coimbra e Leiria responderam a um conjunto de instrumentos administrados no formato de entrevista.

Resultados: 1) A idade, a escolaridade e o local de residência apresentaram uma correlação significativa e no sentido esperado com a saúde física e mental, e com o afeto positivo. O tipo de resposta social dos idosos mostrou-se associado a quase todas as variáveis em estudo. Globalmente, os idosos que se encontram no seu domicílio apresentam uma maior satisfação com a vida, uma melhor saúde física e mental, mais traços compassivos, e mais afeto positivo, comparativamente aos que se encontram sob resposta social; 2) Foram encontradas associações significativas e no sentido esperado entre a autocompaixão, bem-estar subjetivo e estado de saúde; 3) A perceção da saúde física está associada a uma maior satisfação com a vida e menor idade do idoso; a perceção da saúde mental está associada ao aumento da satisfação com a vida, da autocompaixão e diminuição dos afetos negativos; e, por último, a satisfação com a vida está associada a uma superior saúde física e autocompaixão.

Conclusões: Estes resultados sugerem a importância do desenvolvimento de estratégias psicológicas que permitam lidar de forma mais calorosa, tolerante e aceitante o sofrimento resultante dos momentos difíceis típicos da idade avançada, apoiando o possível benefício das terapias focadas na compaixão junto desta população específica, nomeadamente na promoção da satisfação com a vida e saúde mental.
\end{abstract}

\section{Keywords}

Self-compassion

Physical health

Mental health

Subjective well-being

Satisfaction with life

\section{ABSTRACT}

Introduction: The growth in aging population is a society major advance but also entails many challenges, imposing the need for actions promoting successful aging, with higher subjective well-being and better health.

Objectives: To analyse how self-compassion, satisfaction with life, affect, physical and mental health are associated in advanced age, controlling for the effect of sex, age, schooling, place of residence and type of participants' social response. Additionally, this study sought out to explore the variables that better predict satisfaction with life and health in the elderly. Method: The study sample included 155 individuals, aged between 65 and 94 years old, from Coimbra and Leiria districts. Participants completed a set of instruments in an interview format.

Results: 1) Age, schooling and place of residence were significantly correlated in the expected direction with physical and mental health, as well as positive affect. Participants' type of social response was associated with almost all variables. In general, the elderly living at their own home had a higher satisfaction with life, better physical and mental health, more compassionate traits, and more positive affect, when compared to those attending a social response; 2) Significant associations in the expected direction were also found between self-compassion, subjective well-being and health; 3) Greater life satisfaction and younger age were the best predictors of physical health; mental health was best predicted by increased satisfaction with life, self-compassion and decreased negative affect; and, finally, life satisfaction was predicted by a better physical health and by self-compassion.

Conclusions: Results suggest the relevance of developing psychological competencies allowing the elderly to deal with the suffering resulting from difficult/painful moments that may characterize advanced age in a more warmth, tolerant and accepting way. Thus, compassion focused therapies may be beneficial for this specific population aiming the promotion of satisfaction with life and mental health. 


\section{INTRODUÇÃO}

O envelhecimento pode ser definido numa perspetiva geral como um processo progressivo e natural que ocorre em todos os seres vivos de todas as espécies. Em particular no ser humano este processo verifica-se nas dimensões física, psíquica e social. Não ocorre necessariamente de forma simultânea, porém uma dimensão pode interferir, acelerando ou retardando, o processo de uma outra (Becker, 2013; Kanning e Schlicht, 2008). Acredita-se que o envelhecimento resulte do acumular de danos moleculares e celulares ao longo da vida, causados por vários mecanismos e regulados por uma rede complexa de manutenção e reparação (Clegg, Young, Iliffe, Rikkert e Rockwood, 2013).

Cada vez mais se tem assistido a um envelhecimento da população, de ano para ano, com um decréscimo da taxa de natalidade e incremento da esperança de vida. Segundo o Instituto Nacional de Estatística (INE; Carrilho e Craveiro, 2015), em Portugal, a esperança de vida aumentou, entre os triénios de 2001-2003 e 2011-2013, cerca de 3,4 anos para os homens e 2,6 anos para as mulheres, atingindo os 76,9 anos e os 82,9 anos, respetivamente. Portugal foi classificado como o quarto país com maior proporção de pessoas idosas no seio da União Europeia, prevendo-se que este número ultrapasse os 2 milhões. Dados do INE revelam, também, que o valor total da população idosa tem vindo a crescer, sendo em 2001 cerca de $16,5 \%$ do total da população portuguesa, passando para 19,9\% em 2013. Ainda, em 2001, por cada 100 jovens com menos de quinze anos, havia cerca de 103 idosos sendo este rácio aumentado, em 2013, para 136 idosos por cada 100 jovens (Carrilho e Craveiro, 2015).

Embora os números estejam a aumentar, Portugal não tem encontrado as respostas adequadas para esta problemática, posicionando-se como um dos países da Europa Ocidental em que o bem-estar social e económico dos indivíduos com 60 ou mais anos de idade se encontra menos assegurado, sendo apenas superado por Malta e Grécia. A nível mundial (entre os 96 países avaliados) (HelpAge International, 2015), Portugal apresenta-se em $38^{\circ}$ lugar, sendo este ranking liderado pela Suíça, Noruega, Suécia e Alemanha (HelpAge International, 2015).

Segundo a Organização Mundial da Saúde (World Health Organization [WHO], 2002) devem ser adotados, para além dos comportamentos que promovam um envelhecimento saudável, um envelhecimento ativo, ou seja, um processo através do qual seja possível otimizar as oportunidades para a saúde, participação e segurança, e consequentemente melhorar a qualidade de vida das pessoas que envelhecem. Este último conceito é mais abrangente, uma vez que tem em conta, para além da saúde, aspetos socioeconómicos, psicológicos e ambientais (Ribeiro e Paúl, 2011). Perante esta realidade, torna-se cada vez mais pertinente estudar a população de idade avançada, de forma a perceber quais as variáveis significativas modificáveis para que o envelhecimento seja bem-sucedido.

O bem-estar subjetivo diz respeito às avaliações que as pessoas fazem sobre as suas vidas, podendo ser conceptualizado através das seguintes duas componentes: satisfação com a vida (dimensão cognitiva) e experiência de afetos positivos e negativos (dimensão emocional) (Diener, 1984; Guedea et al., 2006; Siqueira e Padovam, 2008). Quanto à componente cognitiva, a satisfação com a vida é a representação mental subjetiva sobre a vida pessoal organizada e armazenada, ou seja, é o julgamento que o indivíduo faz da sua vida e reflete o quão distante ou próximo este se encontra das suas expectativas e desejos tendo em conta o seu estado atual (Pavot e Diener, 1993; Siqueira e Padovam, 2008). Relativamente à dimensão emocional, esta caracteriza-se pelo balanço entre a experiência de emoções positivas (afetos positivos) ou negativas (afetos negativos) na maior parte da vida do indivíduo, ou seja, é a apreciação que o indivíduo faz ao ponderar se a sua vida foi maioritariamente vivenciada através de afetos positivos ou negativos (Pavot e Diener, 1993; Siqueira e Padovam, 2008). Os afetos positivos quando experienciados com elevada intensidade são caracterizados por elevada energia, plena concentração e desempenhos eficientes nas atividades, sendo estas consideradas prazerosas. Já com elevados níveis de afeto negativo, as atividades são realizadas sem prazer, níveis de energia caracterizados por letargia levando o indivíduo a sentir sensações negativas como raiva, desprezo, culpa e nervosismo (Siqueira e Padovam, 2008). Foi corroborado no estudo de Phillips e Ferguson (2013) que a idade se encontrava correlacionada negativamente com os afetos positivos, podendo este decréscimo das emoções positivas dever-se aos múltiplos desafios inerentes ao processo de envelhecimento, tais como o abandono de trabalho devido à reforma, perda do cônjuge e alterações na saúde e/ou funcionamento físico.

No que diz respeito à saúde física e mental, vários são os fatores que podem estar na origem do seu declínio, para além das alterações relacionadas com a idade, surgem os fatores genéticos, doenças e comorbilidades associadas, apoios sociais, de saúde e comportamentos individuais (Organização Mundial da Saúde [OMS], 2015). Muitos dos problemas de saúde surgem diretamente das escolhas mal adaptativas que as pessoas efetuam no quotidiano, não adquirindo comportamentos que promovam a saúde e, em consequência, prolonguem a sua vida. Por exemplo, sabe-se que uma alimentação 
saudável e exercício físico regular estão associados a um baixo risco de doenças coronárias, diabetes e obesidade, não obstante muitos destes parâmetros não serem cumpridos, o que compromete a saúde e aumenta o risco de vir a desenvolver estas doenças (Terry e Leary, 2011).

Dificuldades nos processos de autorregulação, quando relacionadas com comportamentos associados à saúde, podem ter consequências nefastas (Terry e Leary, 2011). Esta competência de conseguir estabelecer objetivos que promovam a saúde, adotar comportamentos para que estes objetivos sejam cumpridos (tais como, procura de tratamento médico adequado e adesão à terapêutica prescrita), monitorizar o progresso e ajustar o comportamento sempre que necessário (Terry e Leary, 2011) são fundamentais para o desenvolvimento de um bom funcionamento físico e mental.

A autocompaixão, enquanto estratégia de regulação emocional, pode, na verdade, encorajar a aquisição destes comportamentos adaptativos, proporcionando uma mudança efetiva no incremento da saúde e do bem-estar (Pinto-Gouveia, Duarte, Matos e Fráguas, 2013). De acordo com Neff (2003a), a autocompaixão pode ser definida segundo três componentes básicos: Capacidade para ser compreensível e amável para consigo próprio, em vez de ser punitivo e crítico (Calor/Compreensão vs. Autocriticismo); entender as suas experiências como parte de uma experiência humana maior (Condição humana vs. Isolamento) e consciência equilibrada dos próprios pensamentos e sentimentos dolorosos, sem uma excessiva sobreidentificação (Mindfulness vs. Sobreidentificação).

Partindo de uma abordagem diferente, com base na psicologia evolucionária e na teoria das mentalidades sociais, Gilbert (2005) e Gilbert e Procter (2006) defendem que a autocompaixão resulta da evolução dos sistemas fisiológicos dos mamíferos relacionados com os comportamentos de vinculação. Por sua vez, o sistema de vinculação pode ser ativado através de dois sinais emitidos pelo comportamento do próprio (interno) como pelo comportamento dos outros (externo) de suporte, bondade, sensibilidade, tolerância na prestação de cuidados, levando a surgirem sentimentos de ligação de calor-afeto e tranquilização. Com efeito, a competência de autocompaixão implica o desenvolvimento de motivação genuína para o cuidado com o bem-estar pessoal, envolvendo ainda a compreensão empática através de uma atitude de não julgamento das experiências de vida (Gilbert, 2005).

Ser autocompassivo implica não ter um padrão de escape ou de evitamento da experiência interna negativa, mas ter o desejo de cuidar do seu sofrimento com calor, compreensão e tolerância (Pinto-Gouveia et al., 2013).
Face a um problema ou uma adversidade, pessoas com uma maior autocompaixão tratam-se a si próprias com preocupação e bondade, em vez de se autocriticarem ou julgarem, reconhecendo essas dificuldades como parte da vida (Brion, Leary e Drabkin, 2014). Assim, quando a pessoa se depara com uma experiência negativa, a autocompaixão pode surgir como um recurso valioso, uma vez que pessoas com elevada autocompaixão têm menor probabilidade de catastrofizar as situações negativas que vão surgindo no decorrer do ciclo vital, experienciando menor ansiedade e não evitando desafios por medo de falhar (Allen e Leary, 2010).

Em amostras na população com idade mais avançada, pode observar-se que a autocompaixão se encontra associada positivamente com a saúde e o bemestar, em geral, satisfação com a vida, função social, envelhecimento bem-sucedido (Allen, Goldwasser e Leary, 2012), saúde mental (Brown, Bryant, Brown, Bei e Judd, 2015), autoestima (Allen e Leary, 2014), afetos positivos, integridade do Ego e sentido da vida (Phillips e Ferguson, 2013). Em sentido contrário, verificou-se associada negativamente com a dor, problemas emocionais, sintomas depressivos (Allen et al., 2012) e afetos negativos (Phillips e Ferguson, 2013).

Idosos com traços mais elevados de autocompaixão apresentavam menos problemas físicos ou reportavam uma melhor saúde em geral, uma maior satisfação com a vida, utilizavam mais frequentemente estratégias de coping adequadas (como, por exemplo, truques de memória e estratégias de assistência) e sentiam-se menos constrangidos pelas suas dificuldades físicas e motoras, em comparação com idosos com baixos níveis de autocompaixão (Allen et al., 2012).

Dadas as possíveis dificuldades físicas e mentais inerentes ao processo de envelhecimento, torna-se pertinente estudar na população idosa portuguesa, quais os possíveis benefícios da autocompaixão no bem-estar subjetivo (satisfação com a vida e afetos positivos e negativos) e na perceção da saúde (física e mental). Assim, os objetivos da presente investigação são: 1) explorar de que modo variáveis como o bem-estar subjetivo (satisfação com a vida e os afetos), o estado de saúde física e mental e a autocompaixão se encontram associados em indivíduos de idade avançada; 2) perceber a relação das variáveis em estudo (autocompaixão, satisfação com a vida, afetos positivos e negativos e perceção do estado de saúde física e mental) com as variáveis sociodemográficas (idade, escolaridade, local de residência); e, 3) explorar qual o conjunto de variáveis que está associado a uma melhor perceção do estado de saúde, física e mental, e a uma maior satisfação com a vida, em indivíduos de idade avançada. 


\section{MÉTODO}

\section{Procedimentos}

Inicialmente foram recolhidas as autorizações dos autores dos instrumentos que seriam necessários para o presente estudo. Posteriormente foi construído o protocolo de avaliação constituído pela folha de rosto, questionário sociodemográfico e pelos instrumentos psicológicos de autorrelato, no qual eram explicados os objetivos do estudo e as questões éticas inerentes, tais como, a confidencialidade, participação voluntária, possibilidade de desistência em qualquer momento e anonimato, sendo os dados recolhidos apenas para fins estatísticos. De seguida, foram solicitadas as autorizações aos diretores das instituições onde foi recolhida a amostra, sendo-lhes explicado os objetivos, metodologias adotadas na investigação, assim como a finalidade dos dados obtidos.

Relativamente à recolha de dados, apesar da utilização de questionários de autorrelato, estes foram aplicados no formato de entrevista uma vez que existiram algumas condicionantes, tais como, por exemplo, escolaridade muito baixa e défice de acuidade visual. Assim, a avaliação decorreu individualmente, num contexto calmo e privado, tendo sido explicados aos participantes os objetivos, metodologias e finalidades do estudo, bem como pedido o seu consentimento informado. As entrevistas foram realizadas pela primeira autora ao longo de 6 meses, em instituições de conveniência que proporcionam cuidados à terceira idade, nomeadamente numa unidade incluída na Rede Nacional de Cuidados Continuados Integrados (RNCCI), em três estruturas residenciais para idosos (ERPI) (dois dos quais com centro de dia integrado), dois centros de dia, bem como nos domicílios de idosos que se encontravam sem qualquer resposta social institucional, dos distritos de Leiria e Coimbra, zona centro do país.

\section{Amostra}

A amostra inicial deste estudo foi constituída por 193 participantes dos quais apenas 155 (79\%) foram considerados. Os restantes 38 casos (21\%) foram excluídos uma vez que não cumpriam os seguintes critérios de inclusão: a) Idade igual ou superior a 65 anos de idade; b) Ausência de doença aguda; c) Ausência de demência e défice $\operatorname{cognitivo~}^{1}$; d) Cedência do consentimento informado.

A amostra final foi constituída por 99 mulheres $(63,9 \%)$ e 56 homens $(36,1 \%)$, com idades compreendidas entre os 65 e os 94 anos, sendo a média de idades de 76,06 $(D P=7,15)$. Relativamente à

\footnotetext{
1 Para averiguar este critério, foi utilizado o teste de rastreio cognitivo, o MoCA, tendo como pontes corte as seguintes pontuações: indivíduos com escolaridade entre os 0 - 4 anos $(M=21,71 ; D P=3,37)$; indivíduos com escolaridade entre os 5 - 9 anos $(M=24,60 ; D P=2,87)$; indivíduos
}

escolaridade, apresentaram uma média de 3,50 (DP = 2,91 ) anos de estudo, sendo que a sua maioria indicou uma escolaridade entre 1 a 4 anos de escolaridade (74, $2 \%$ ). No que toca ao meio de residência, 128 participantes habitavam em meio rural $(82,6 \%)$ e 27 em meio urbano $(17,4 \%)$.

\section{Instrumentos}

Questionário sociodemográfico. Desenvolvido pela equipa de investigação, pretende recolher informação relativa aos dados sociodemográficos, tais como, a idade, o sexo, o meio de residência, os anos de escolaridade e a tipologia de institucionalização ou resposta social.

Avaliação Cognitiva de Montreal (MoCA, Nasreddine et al., 2005; Versão Portuguesa: Freitas, Simões, Martins, Vilar, Santana, 2010). Este teste foi utilizado com o intuito de realizar um rastreio cognitivo para a deteção do défice cognitivo ligeiro (DCL). Avalia os seguintes domínios cognitivos: Orientação (6 pontos), memória a curto prazo (Evocação, 5 pontos), capacidade visuoespacial (desenho do relógio, 3 pontos; cópia do cubo, 1 ponto), função executiva (tarefa de trilhas, 1 ponto), abstração verbal (2 pontos), fluência fonémica (1 ponto), linguagem (nomeação de animais, 3 pontos; repetição de duas frases, 2 pontos) e atenção, concentração e memória operatória (deteção de uma letra, utilizando o toque, 1 ponto; subtração em série, 3 pontos; sequência de números em sentido direto e inverso, 2 pontos). Na versão portuguesa, a escala revelou uma consistência interna razoável ( $\alpha$ de Cronbach de 0,78 ), sendo que no presente estudo esta se manteve ( $\alpha$ de Cronbach de 0,76).

Escala da Autocompaixão (Self-Compassion Scale SCS, Neff, 2003b; Versão Portuguesa: Castilho e Gouveia, 2011). A versão original desta escala foi desenvolvida por Neff (2003b) com o objetivo de medir o constructo da autocompaixão. Esta escala é composta por 26 itens distribuídos por seis subescalas distintas: Calor/compreensão, Autocrítica, Humanidade comum, Isolamento, Mindfulness e Sobreidentificação. O formato de resposta é tipo Likert de 5 pontos, variando entre 1 (Quase nunca) e 5 (Quase sempre), sendo que quanto maior for a pontuação obtida, maior será a autocompaixão, resultando da soma das subescalas, depois de invertidas as subescalas de valência negativa. $\mathrm{Na}$ versão portuguesa, a escala obteve uma boa consistência interna ( $\alpha$ de Cronbach de 0,89) e estabilidade temporal (Castilho e Gouveia, 2011). No presente estudo, à semelhança de outras investigações sobre a compaixão em idosos, apenas será utilizado o

com escolaridade entre os $10-12$ anos $(M=25,11$; $D P=1,94)$ e, por último, indivíduos com escolaridade $>12$ anos $(M=26,35 ; D P=1,87)$ (Freitas, Simões, Alves e Santana, 2011). 
total deste instrumento, revelando este uma boa consistência interna ( $\alpha$ de Cronbach de 0,80 ).

Questionário do estado de saúde (Short Form Health Survey - 36 Item - SF-36v2 (versão 2); Ware e Sherbourne, 1992; Versão Portuguesa: Ferreira, 2000). Este questionário avalia o estado de saúde de indivíduos em diferentes faixas etárias, monitoriza doentes com diversas condições clínicas e ainda compara estes com a população geral. Na versão original é constituído por 36 itens que são agrupados em oito dimensões: função física, desempenho físico, dor física, saúde geral, vitalidade, função social, desempenho emocional e saúde mental. Estas oito dimensões podem ser agrupadas em duas componentes principais: saúde física e saúde mental (Ferreira, 2000; Ware e Sherbourne, 1992). A pontuação da escala tem uma orientação positiva, ou seja, quanto maior a pontuação obtida pelo indivíduo, melhor será o seu estado de saúde percecionado, podendo variar entre 0 e 100 pontos, para cada componente (Ferreira, 2000). Foi efetuada uma validação mais abrangente, tendo obtido valores de alfa de Cronbach de 0,86 (componente física) e 0,87 (componente mental), sendo definidas medidas sumárias para a população geral portuguesa (Ferreira, Ferreira e Pereira, 2012). No presente estudo, a escala apresenta uma consistência interna muito boa ( $\alpha$ de Cronbach de 0,90, componente física e componente mental de 0,89).

Escala de Satisfação com a Vida (Satisfaction With Life Scale - SWLS; Diener Emmons, Larsen e Griffin, 1985; Versão Portuguesa: Simões, 1992). Esta escala avalia a forma como os indivíduos experienciam a sua vida. A versão portuguesa é constituída por cinco itens, formulados no sentido positivo, com uma escala de resposta tipo Likert de um a cinco pontos, podendo a pontuação variar entre cinco e 25 pontos, com um ponto médio de 15 pontos, sendo que quanto maior for a pontuação obtida, maior será a satisfação com a vida. A escala obteve, no estudo para validação na população portuguesa, um coeficiente de alfa de Cronbach de 0,77 e revelou uma estrutura unifatorial (Simões, 1992). No presente estudo, a escala revela uma boa consistência interna, com um alfa de Cronbach de 0,80.

Escala de Afeto Positivo e Negativo (Positive and Negative Affect Schedule - PANAS; Watson, Clark e Tellegen, 1988; Versão Reduzida Portuguesa: Galinha, Pereira e Esteves, 2014). Esta escala avalia a vertente afetiva do bem-estar subjetivo. A versão portuguesa é constituída por 20 emoções divididas nas subescalas de afeto positivo e afeto negativo, através de uma escala tipo Likert. Em termos de consistência interna, esta demonstra-se adequada com valores de 0,86 para a escala de afeto positivo e de 0,89 para a escala de afeto negativo (Galinha e Pais-Ribeiro, 2005). Neste estudo será utilizada a versão portuguesa reduzida que foi desenvolvida a partir da versão anteriormente descrita. Esta é constituída por 10 itens divididos em duas escalas: afeto positivo e afeto negativo, podendo a escala de resposta variar entre um (Nada ou muito ligeiramente) e cinco (Extremamente). Revela boas características psicométricas e uma correlação elevada com a versão de 20 itens (Galinha et al., 2014). No presente estudo, a escala dos afetos positivos apresenta um alfa de Cronbach de 0,82 , sendo que para os afetos negativos apresenta um alfa de Cronbach de 0,79.

\section{Análise estatística}

No presente estudo, para o tratamento estatístico foi utilizado o software Statistical Package for the Social Sciences (SPSS), versão 20.

A análise da consistência interna dos instrumentos foi efetuada através do Alfa de Cronbach. Para analisar as associações entre as diversas variáveis, utilizou-se o coeficiente de correlação de Pearson para variáveis contínuas e correlação bisserial por pontos para analisar a associação entre uma variável contínua e variável com duas categorias (sexo e meio de residência). A interpretação da magnitude das associações baseou-se nas recomendações de Pestana e Gageiro (2008) (associação muito baixa: $r<0,2$; associação baixa: $0,2 \leq$ $r \leq 0,39$; associação moderada: 0,4 $\leq r \leq 0,69$; associação alta: $0,7 \leq r \leq 0,89$; associação muito alta: $0,9 \leq r \leq 1)$.

O pressuposto da distribuição normal das variáveis foi explorado através do teste de Kolmogorov-Smirnov e dos valores de assimetria (sk) e de achatamento (ku). Os valores de sk e ku não foram indicadores da existência de violações severas a este pressuposto $(|s k|<3$ e $|k u|<$ 10) (Kline, 1998). Relativamente ao pressuposto da homogeneidade da variância, validado através do teste de Levene, não se observou um valor significativo em nenhuma das variáveis consideradas.

A comparação dos valores médios das variáveis em estudo, em função da resposta social, foi feita através de uma ANOVA one-way, com recurso aos testes post hoc HSD de Tukey para localizar as diferenças significativas entre os grupos.

Por último, foram realizadas análises de regressão linear múltiplas a fim de analisar o conjunto de variáveis que melhor prediz o estado de saúde, física e mental, e a satisfação com a vida. Neste contexto procedeu-se à verificação da independência dos erros através da análise gráfica da distribuição dos dados e dos valores de Durbin-Watson, bem como dos valores da Variance Inflaction Factor (VIF), os quais se mostraram inferiores a cinco.

Valores de prova inferiores ou iguais a 0,05 foram considerados indicadores de significância estatística. 


\section{RESULTADOS}

\section{Correlação entre o bem-estar subjetivo, a perceção do estado de saúde e a autocompaixão}

Na Tabela 1 é apresentada a matriz de correlações de Pearson entre as variáveis bem-estar subjetivo, perceção do estado de saúde e autocompaixão.

Tabela 1

Análise das correlações entre o bem-estar subjetivo, a perceção do estado de saúde e a autocompaixão $(N=155)$

\begin{tabular}{|c|c|c|c|c|c|c|}
\hline & & \multirow{2}{*}{ SV } & \multicolumn{2}{|c|}{ Afetos } & \multicolumn{2}{|c|}{ PES } \\
\hline & & & Negativo & Positivo & Física & Mental \\
\hline \multicolumn{2}{|c|}{$\begin{array}{l}\text { Satisfação com a Vida } \\
\text { (SV) }\end{array}$} & - & & & & \\
\hline \multirow{2}{*}{ Afetos } & Negativos & $0,45^{* *}$ & - & & & \\
\hline & Positivos & $0,29^{* *}$ & $-0,20$ & & & \\
\hline \multirow{2}{*}{$\begin{array}{l}\text { Perceção do } \\
\text { Estado de } \\
\text { Saúde (PES) }\end{array}$} & Física & $0,52^{* *}$ & $-0,37^{* *}$ & 0,16 & - & \\
\hline & Mental & $0,61^{* *}$ & $-0,62^{* *}$ & $0,33^{* *}$ & $0,69^{* *}$ & - \\
\hline \multicolumn{2}{|c|}{ Autocompaixão } & $0,50^{* *}$ & $-0,51^{* *}$ & $0,19^{* *}$ & $0,35^{* *}$ & $0,61^{* *}$ \\
\hline
\end{tabular}

De acordo com a Tabela 1, podemos verificar que a satisfação com a vida apresenta uma associação significativa moderada negativa com os afetos negativos e uma correlação baixa positiva com os afetos positivos. Esta variável encontra-se associada moderadamente, de forma positiva e estatisticamente significativa, com a perceção da saúde física e saúde mental. Por sua vez, encontra-se correlacionada moderadamente, de forma positiva e estatisticamente significativa, com a autocompaixão.

Relativamente à perceção do estado de saúde física verifica-se uma associação baixa positiva com a autocompaixão e uma correlação baixa negativa com os afetos negativos.

Já na componente da perceção da saúde mental, esta demonstra as seguintes associações: correlacionada moderadamente, de forma negativa, com os afetos negativos e com uma baixa associação positiva, estatisticamente significativa, com os afetos positivos e moderadamente de forma positiva e significativa com a autocompaixão.

Quanto à autocompaixão pode observar-se que apresenta uma correlação moderada negativa, estatisticamente significativa, com os afetos negativos e uma associação muito baixa positiva com os afetos positivos.
Estudo da associação entre a idade, escolaridade, sexo, meio de residência e tipo de resposta social e as variáveis em estudo (bem-estar subjetivo, perceção do estado de saúde e autocompaixão)

A idade revelou uma associação negativa baixa com a perceção da saúde física $(r=-0,215 ; p=0,007)$ e com o afeto positivo $(r=-0,279 ; p<0,001)$, e correlação negativa muito baixa com a perceção da saúde mental $(r$ $=-0,178 ; p=0,027$ ). A idade não se mostrou associada à autocompaixão, à satisfação com vida e ao afeto negativo.

A escolaridade evidenciou apenas uma associação positiva baixa com o afeto positivo $(r=0,286 ; p<0,001)$ e uma correlação muito baixa, mas ainda significativa, com a perceção da saúde mental $(r=0,170 ; p=0,034)$.

O sexo não se mostrou associado a nenhuma variável em estudo (autocompaixão, perceção do estado de saúde e bem-estar subjetivo).

Quanto ao meio de residência, são os idosos que vivem em meio rural que apresentam valores mais elevados na perceção do estado de saúde física ( $M=$ $62,95 ; D P=12,91)$, comparativamente com idosos residentes em meio urbano $(M=53,21 ; D P=16,47)$, sendo esta diferença estatisticamente significativa $[t(1,153)=$ $3,39 ; p=0,001]$. Não foram encontradas diferenças significativas para as demais variáveis em estudo.

Quando comparados os valores médios das diversas variáveis em função do tipo de resposta social, verificamse diferenças significativas em todas as variáveis, à exceção do afeto negativo (Tabela 2). Estas diferenças ocorrem entre os idosos sem resposta social, comparativamente àqueles que se encontram em centro de dia, ERPI e RNCCl, apresentando os primeiros valores mais elevados na perceção do estado de saúde física (centro de dia: $p=0,014$; ERPI: $p<0,001$; RNCCI: $p<$ $0,001)$. Pode ainda analisar-se, nesta componente, que os idosos que se encontram no centro de dia apresentam valores mais elevados quando comparados com os que estão institucionalizados em ERPI $(p=0,037)$ ou na $\operatorname{RNCCl}(p<0,001)$.

Quanto à perceção da saúde mental, pode verificarse uma diferença estatisticamente significativa, na qual os idosos que não se encontram institucionalizados, apresentam valores mais elevados quando comparados com aqueles que se encontram no centro de dia $(p=$ $0,022)$ e na $\mathrm{RNCCl}(p<0,001)$.

Relativamente à autocompaixão, os idosos que não se encontram sob resposta social apresentam valores mais elevados, quando comparados com aqueles que frequentam os centros de dia $(p=0,048)$.

Observou-se que os idosos sem resposta social institucional apresentavam valores mais elevados na satisfação com a vida, quando comparados com os utentes da $\operatorname{RNCCI}(p=0,020)$ e da $\operatorname{ERPI}(p=0,004)$. 
Tabela 2

Variáveis em estudo (satisfação com a vida, estado de saúde e bem-estar subjetivo) em função do tipo de resposta social (sem resposta social, centro de dia, ERPI e RNCCI) $(N=155)$

\begin{tabular}{|c|c|c|c|c|c|c|c|c|c|c|c|}
\hline & & \multicolumn{2}{|c|}{$\begin{array}{l}\text { Sem resposta } \\
\text { social }\end{array}$} & \multicolumn{2}{|c|}{ Centro de dia } & \multicolumn{2}{|c|}{ ERPI } & \multicolumn{2}{|c|}{$\mathrm{RNCCI}$} & \multirow{2}{*}{$\begin{array}{c}\boldsymbol{F} \\
(3,151)\end{array}$} & \multirow{2}{*}{$p$} \\
\hline & & $M$ & $D P$ & $M$ & $D P$ & $M$ & $D P$ & $M$ & $D P$ & & \\
\hline \multicolumn{2}{|c|}{ Satisfação com a Vida } & 18,91 & 4,21 & 16,89 & 5,01 & 14,74 & 5,75 & 15,13 & 6,03 & 6,25 & 0,001 \\
\hline \multirow{2}{*}{ Estado de Saúde } & Físico & 67,13 & 10,68 & 59,59 & 11,99 & 50,38 & 13,22 & 43,30 & 12,27 & 27,29 & $<0,001$ \\
\hline & Mental & 56,92 & 8,51 & 50,93 & 9,59 & 51,00 & 11,26 & 43,31 & 13,05 & 10,99 & $<0,001$ \\
\hline \multicolumn{2}{|l|}{ Autocompaixão } & 94,63 & 11,90 & 87,82 & 10,51 & 89,89 & 14,18 & 93,31 & 13,11 & 2,66 & 0,050 \\
\hline \multirow{2}{*}{ Afetos } & Positivos & 14,30 & 3,58 & 11,64 & 3,97 & 12,26 & 5,67 & 14,75 & 4,49 & 4,24 & 0,007 \\
\hline & Negativos & 7,47 & 3,06 & 9,07 & 3,77 & 8,26 & 3,28 & 8,88 & 3,63 & 2,22 & 0,088 \\
\hline
\end{tabular}

Notas: ERPI - Estrutura Residencial para Idosos; RNCCI - Unidade Integrada na Rede Nacional de Cuidados Continuados Integrados.

Quanto ao afeto positivo, verificou-se que os indivíduos sem resposta social institucional apresentam valores mais elevados quando comparados com aqueles que frequentam os centros de dia $(p=0,014)$. Não se verificaram diferenças estatisticamente significativas em relação ao afeto negativo.

\section{Análises de regressão linear múltipla}

Foram realizadas análises de regressão linear múltipla para compreender quais as variáveis que contribuem para uma melhor perceção do estado de saúde, física e mental, e para uma maior satisfação com a vida.

Para predizer uma melhor perceção do estado de saúde física e saúde mental, foram utilizadas como variáveis independentes: satisfação com a vida, autocompaixão, afetos (positivos e negativos) e a idade, uma vez que esta variável se mostrou associada ao estado de saúde global.

Para a satisfação com a vida, foram utilizadas as seguintes variáveis independentes: perceção do estado de saúde (física e mental), autocompaixão e afetos (positivos e negativos).

No caso da perceção do estado de saúde física (Tabela 3), o modelo é significativo $\left[R^{2}=0,32 ; F(5,149)=\right.$ 14,04; $p<0,001$ ], explicando $32 \%$ da variância. Emergem como preditores a satisfação com a vida ( $\beta=$ $0,41 ; p<0,001)$ e a idade $(\beta=-0,17 ; p=0,016)$.

Relativamente à perceção do estado de saúde mental, o modelo (Tabela 3) demonstrou-se significativo $\left[R^{2}=0,59 ; F(5,149)=42,53 ; p<0,001\right]$, explicando $59 \%$ da variância. Neste modelo é possível observar que surgem como variáveis preditoras os afetos negativos $(\beta=$ $-0,32 ; p<0,001)$, a satisfação com a vida $(\beta=0,28 ; p<$ $0,001)$ e a autocompaixão $(\beta=0,28 ; p<0,001)$.
Tabela 3

Análise de regressão múltipla utilizando as variáveis satisfação com a vida, autocompaixão, afetos e idade para predizer a perceção do estado de saúde física e mental em idosos $(N=155)$

\begin{tabular}{|c|c|c|c|c|c|c|c|}
\hline & & \multicolumn{6}{|c|}{ Perceção do estado de saúde } \\
\hline & & \multicolumn{3}{|c|}{ Física } & \multicolumn{3}{|c|}{ Mental } \\
\hline & & $\mathbf{R}^{2}$ & $\beta$ & $p$ & $\mathbf{R}^{2}$ & $\boldsymbol{\beta}$ & $p$ \\
\hline & & 0,32 & & $<0,001$ & 0,59 & & $<0,001$ \\
\hline \multicolumn{2}{|c|}{ Satisfação com a Vida } & \multicolumn{3}{|c|}{$0,41<\mathbf{0 , 0 0 1}$} & \multicolumn{3}{|c|}{$0,28<\mathbf{0 , 0 0 1}$} \\
\hline \multicolumn{2}{|c|}{ Autocompaixão } & \multicolumn{2}{|r|}{0,07} & 0,413 & \multicolumn{3}{|c|}{$0,28<\mathbf{0 , 0 0 1}$} \\
\hline \multirow{2}{*}{ Afetos } & Negativos & & $-0,15$ & 0,074 & \multirow{2}{*}{\multicolumn{3}{|c|}{$\begin{array}{r}-0,32<\mathbf{0 , 0 0 1} \\
0.110,057\end{array}$}} \\
\hline & Positivos & & 0,05 & 0,459 & & & \\
\hline \multicolumn{2}{|l|}{ Idade } & & $-0,17$ & 0,016 & & $-0,07$ & 0,185 \\
\hline
\end{tabular}

Por último, em relação à satisfação com a vida, o modelo (Tabela 4) mostrou ser significativo $\left[R^{2}=0,44 ; F(5\right.$, 149) $=23,15 ; p<0,001$, explicando $44 \%$ da variância. Como preditores significativos surgem a perceção do estado de saúde física $(\beta=0,24 ; p=0,006)$ e a autocompaixão $(\beta=0,22 ; p=0,004)$.

\section{Tabela 4}

Análise de regressão múltipla utilizando as variáveis perceção do estado de saúde, autocompaixão e afetos para predizer a satisfação com a vida em idosos $(N=155)$

\begin{tabular}{llccc}
\hline & & \multicolumn{3}{c}{ Satisfação com a vida } \\
\cline { 3 - 4 } & & $\mathbf{R}^{2}$ & $\boldsymbol{B}$ & $\boldsymbol{p}$ \\
\cline { 3 - 4 } & & 0,44 & & $<0,001$ \\
\hline Perceção do estado & Física & 0,24 & $\mathbf{0 , 0 0 6}$ \\
de saúde & Mental & 0,21 & 0,072 \\
\hline Autocompaixão & & 0,22 & $\mathbf{0 , 0 0 4}$ \\
\hline \multirow{2}{*}{ Afetos } & Negativos & $-0,09$ & 0,253 \\
& Positivos & 0,12 & 0,063 \\
\hline
\end{tabular}




\section{DISCUSSÃO}

O presente estudo teve como objetivo principal explorar de que modo variáveis como o bem-estar subjetivo (satisfação com a vida e os afetos), o estado de saúde física e mental e a autocompaixão se encontram associados em indivíduos de idade avançada. Adicionalmente, pretendeu-se analisar a relação entre as variáveis em estudo e variáveis sociodemográficas como o sexo, a idade, a escolaridade, o local de residência e o tipo de resposta social. Por último, procurou-se examinar quais as variáveis que se constituem como preditores da satisfação com a vida e da saúde física e mental nesta população.

Em relação ao primeiro estudo, os resultados apontam para a existência de associações positivas e significativas entre o bem-estar subjetivo e a saúde física e mental e a autocompaixão. Por sua vez, a ocorrência de correlações negativas verificou-se apenas quando considerado o afeto negativo e as demais variáveis. Este padrão de resultados foi também reportado em estudos anteriores, conduzidos em amostras da população idosa, os quais demonstraram que a autocompaixão se associa, não só ao bem-estar subjetivo, mas também ao estado de saúde (física e mental) (Allen et al., 2012; Brown et al.,2015; Phillips e Ferguson, 2013; Pinto-Gouveia et al., 2013). De facto, as competências autocompassivas permitem aos idosos perceber as suas dificuldades inerentes ao processo de envelhecimento de forma calorosa e compreensiva, moderando as reações perante acontecimentos indutores de stresse e adquirindo iniciativa para lidar com as suas falhas, o que conduz à adoção de um estilo de coping adaptativo perante as adversidades (Allen e Leary, 2010; Allen et al., 2012). Diversos estudos indicam que, em momentos de sofrimento e dor, tratarmo-nos a nós próprios e aos outros com gentileza e compreensão, percebendo a dor ou o fracasso como parte de uma experiência humana mais ampla, proporciona um bem-estar subjetivo, intrapessoal, interpessoal e físico (Allen et al., 2012; Hall, Row, Wuensch e Godley, 2013; Neff e Costigan, 2014; Phillips e Ferguson, 2013). Na globalidade, pode dizer-se que a autocompaixão tem sido associada positivamente ao bem-estar em diferentes etapas do ciclo de vida, sendo esta associação reportada em múltiplos estudos (Allen et al., 2012; Hall et al., 2013; Neely, Schallert, Mohammed, Roberts e Chen, 2009; Phillips e Ferguson, 2013). Por sua vez, tanto o bem-estar subjetivo como a saúde, física e mental, têm vindo a ser apontados como elementos chave para um envelhecimento bem-sucedido (Allen et al., 2012; Phillips e Ferguson, 2013).

No que concerne ao segundo estudo, da relação entre as variáveis em estudo e as variáveis sociodemográficas, constatou-se não existir relação entre o sexo e as variáveis consideradas. Já no que toca à idade, observou-se que os sujeitos mais velhos percecionavam a sua saúde física e mental como mais deficitária e apresentavam menos afetos positivos. Por sua vez, no que respeita à escolaridade, os indivíduos com níveis de escolaridade mais elevados evidenciavam mais afeto positivo e melhor saúde mental. Quando considerado o meio de residência, apenas a perceção do estado de saúde física se revela significativamente melhor nos sujeitos residentes em meio rural. Relativamente à resposta social, os indivíduos sem resposta social apresentaram valores superiores em todas as variáveis, à exceção dos afetos negativos.

A inexistência de associações entre o sexo e as variáveis estudadas deverá ser interpretada com prudência. De notar que a amostra do presente estudo apresenta um predomínio de participantes do sexo feminino, o que poderá ter contribuído para os resultados obtidos. Para além disso, parece não existir consenso na literatura no que respeita à influência exclusiva do sexo em variáveis como a autocompaixão, a perceção do estado de saúde e o bem-estar subjetivo. A título de exemplo, Phillips e Ferguson (2013), tal como sucedeu no nosso estudo, também não reportaram a existência de diferenças entre homens e mulheres de idade avançada relativamente à autocompaixão. No entanto, mais recentemente, e no que concerne a diferenças entre homens e mulheres relativamente à autocompaixão ao longo do ciclo de vida, a metanálise conduzida por Yarnell et al. (2015) indicou que os homens apresentavam níveis ligeiramente mais altos, ainda que com um tamanho do efeito de pequena dimensão.

Quanto à idade, verificou-se que quanto maior a idade, pior é a perceção do estado de saúde físico e menos afetos positivos são sentidos, o que se pode dever aos múltiplos desafios inerentes ao processo de envelhecimento, tais como alterações nos papéis sociais até ao momento desempenhados (por ex., reforma), perda do cônjuge e/ou amigos próximos e mudanças na saúde e funcionamento físico (por ex., alterações cognitivas, mudanças no sistema endócrino, no sistema imunológico e no sistema músculo-esquelético; perda nas reservas fisiológicas cardiovasculares, respiratórias e renais e estado nutricional) (Clegg et al., 2013; OMS, 2015; Phillips e Ferguson, 2013).

Observou-se, no presente estudo, que quanto maior a escolaridade, melhor é a saúde mental e a frequência de afetos positivos. Este dado sugere que a escolaridade pode exercer um papel protetor no envelhecimento cognitivo e na afetividade o que está de acordo com dados reportados em alguns estudos (Burns, Anstey e Windsor, 2011; Falcão, Espírito Santo, Matreno, Fermino e Guadalupe, 2012). Contudo, este mecanismo não se encontra completamente esclarecido e deverá ser alvo de 
investigação mais aprofundada, uma vez que a investigação tem produzido resultados mistos, apontando a interveniência de outras variáveis que influenciam esta ligação (Ardila, Ostrosky-Solis, Rosselli e Gómez, 2000).

Em relação à ligação entre o meio de residência e a autocompaixão, bem-estar subjetivo e estado de saúde, verificou-se que os idosos que habitam em meio rural apresentam valores mais elevados na saúde física, quando comparados com os idosos em meio urbano. Estes achados vão ao encontro do modelo ecológico de Lawton (1983), que perspetiva que o contexto de residência é essencial para a compreensão do processo de envelhecimento e o facto de este ser ou não bemsucedido. Defende ainda que muitos dos comportamentos adotados são definidos pelas competências que o idoso apresenta em relação à pressão exercida pelo meio ambiente, ou seja, são vistas perante um determinado potencial de exigência. De forma global, num meio de residência rural é fomentada menos pressão sobre os idosos, o que leva a que vivam em maior consonância com o ambiente (Lawton, 1989). Ainda, os idosos residentes em meio rural e não institucionalizados, relataram frequentemente ao longo da recolha dos dados, que continuam a cuidar dos seus animais, das fazendas e terras de cultivo, o que contribui para que permaneçam ativos. Efetivamente, estudos anteriores indicam que a manutenção das atividades e rotinas habituais desempenham um importante papel na promoção da saúde e bem-estar (Sequeira e Silva, 2002; Teixeira, 2010).

No que respeita à variável resposta social, verificouse que os idosos que ainda não se encontram institucionalizados apresentam valores mais elevados, e significativamente relevantes, na saúde física, na saúde mental, autocompaixão e afeto positivo, quando comparados com os idosos institucionalizados (centro de dia, RNCCl e lar). De alguma forma estes resultados eram expectáveis, uma vez que os estudos indicam que a institucionalização está associada a baixos níveis de atividade física, de estimulação cognitiva e, consequentemente, uma menor funcionalidade global (Del Duca, Silva, Thumé, Santos e Hallal, 2012; Pinto, 2013; Vitorino, Paskulin e Vianna, 2013). Contudo, recorrentemente esta institucionalização é das poucas soluções que as famílias possuem para que os cuidados de saúde aos seus familiares sejam assegurados (Pinto, 2013), quer seja numa tipologia apenas diurna, como os centros de dia, internamentos temporários, como a $\mathrm{RNCCl}$, quer internamentos a longo prazo, como as estruturas residenciais para idosos. Outra realidade também recorrente é o facto de os idosos, aquando da entrada nas instituições, já apresentarem quadros clínicos prévios, sendo esse o motivo da sua institucionalização, requerendo cuidados permanentes. $\mathrm{Na}$ amostra institucionalizada, pode verificar-se uma associação pertinente, em que os idosos que se encontram em centro de dia apresentam valores mais elevados no estado de saúde física quando comparados com aqueles que se encontram na RNCCl e lar. Estes dados podem dever-se ao facto de que os idosos que se encontram em centro de dia, não necessitarem de permanecer internados, regressando ao domicílio no final do dia. Pressupõe-se que ao regressarem a casa consigam retomar algumas das suas atividades valorizadas, permanecendo mais ativos e mentalmente estimulados, do que aqueles que se encontram internados ( $\mathrm{RNCCl}$ e lar), sabendo-se que quanto mais ativos, melhor será o estado de saúde e mais bem-sucedido será o envelhecimento (Ribeiro e Paúl, 2011; WHO, 2002).

No que diz respeito ao modelo preditivo do estado de saúde físico, surgiram como variáveis significativas a satisfação com a vida e a idade. Neste contexto, poderse-á hipotetizar que a apreciação que os idosos fazem das suas vidas, que reflete o quão distantes se encontram das suas expectativas e desejos, poderá influenciar a perceção que têm da sua saúde física. Também a idade pode influenciar esta componente da saúde de forma negativa, ou seja, à medida que se vai envelhecendo, a probabilidade de surgirem problemas de saúde aumenta (Allen et al., 2012; Clegg et al., 2013; Phillips e Ferguson, 2013) sendo este um aspeto considerado na própria definição de envelhecimento (Becker, 2013; Clegg et al., 2013; Kanning e Schlicht, 2008).

Por sua vez, o estado de saúde mental subjetiva foi melhor predito pela satisfação com a vida, pelos afetos negativos e pela autocompaixão. Este resultado sugere que a satisfação com a vida, o encarar com calor e compreensão os acontecimentos menos bons, percebendo que estes fazem parte da uma experiência humana alargada, sem se sobreidentificarem excessivamente com os mesmos, são elementos importantes para que os idosos se percecionem como detentores de uma boa saúde mental (Neff, 2003a). Também a experiência de afetos negativos representa um papel fundamental nesta componente da saúde, tendose verificado que menores níveis de emoções negativas, tendem a associar-se a uma melhor perceção da saúde mental (Clegg et al., 2013; Diener, 1984).

A satisfação com a vida revelou ter um contributo relevante, tanto para a saúde física, como para a saúde mental, pelo que se torna então pertinente perceber o que pode predizer a mesma. Assim, no modelo preditivo da satisfação com a vida em idosos, surgiram como variáveis significativas a saúde física e a autocompaixão. Desta forma, valores mais elevados de autocompaixão e um estado de saúde física mais favorável estão associados a uma melhor apreciação que os idosos fazem das suas vidas. 
Algumas limitações devem ser apontadas na interpretação dos resultados obtidos. Com efeito, o desenho transversal do presente estudo invalida o estabelecimento de relações causais entre as variáveis estudadas. De mencionar, também, que o modo como os dados foram recolhidos, em formato de entrevista, devido a algumas dificuldades apresentadas pelos idosos, poderá ter influenciado as respostas no sentido da desejabilidade social. A amostra verificou-se relativamente reduzida, maioritariamente recolhida em meio rural e com idosos com baixa escolaridade, sendo desejável, em estudos posteriores, atender a estas questões para generalização dos resultados. Pode ainda ser apontado o facto da variável saúde ter sido avaliada sem consulta de processos médicos, baseando-se apenas nas perceções que estes apresentam da sua saúde mental e física.

Não obstante as limitações apontadas, os resultados sugerem direções para investigação futura e implicações práticas para o desenvolvimento de intervenções de caráter psicossocial dirigidas à população com idade avançada. Este estudo propõe pistas de reflexão para a criação e aplicação de estratégias e programas integrados de intervenção que visem o desenvolvimento de competências autocompassivas, considerando que estas poderão conduzir a uma maior satisfação com a vida, melhor saúde mental e física em idosos. Atendendo ao inequívoco fenómeno do envelhecimento populacional à escala mundial (HelpAge International, 2015; OMS, 2015) e à reconhecida necessidade de melhoria das respostas para esta faixa etária em Portugal (Carrilho e Craveiro, 2015; HelpAge International, 2015), são particularmente importantes os contributos para o desenvolvimento de intervenções cujas componentes se tenham mostrado empiricamente relevantes.

Conflito de interesses | Conflict of interest: nenhum | none.

Fontes de financiamento | Funding sources: nenhuma | none.

\section{REFERÊNCIAS}

Allen, A. B. e Leary, M. R. (2010). Self-compassion, stress, and coping. Social and Personality Psychology Compass, 4(2), 107-118. doi:10.1111/j.1751-9004.2009.00246.x

Allen, A. B., Goldwasser, E. R. e Leary, M. R. (2012). Self-compassion and well-being among older adults. Self and Identity, 11(4), 428453. doi:10.1080/15298868.2011.595082

Allen, A. B. e Leary, M. R. (2014). Self-compassionate responses to aging. The Gerontologist, 54(2), 190-200. doi:10.1093/geront/gns204

Ardila, A., Ostrosky-Solis, F., Rosselli, M. e Gómez, C. (2000). Agerelated cognitive decline during normal aging: The complex effect of education. Archives of Clinical Neuropsychology, 15(6), 495513. doi:10.1016/S0887-6177(99)00040-2

Becker, A. M. (2013). Exercício físico, qualidade de vida e autoestima global em idosos portugueses: Um estudo exploratório do instrumento Whoqol-old (Dissertação de mestrado, Universidade de Coimbra, Coimbra). Disponível em http://hdl.handle.net/10316/25068
Brion, J. M., Leary, M. R. e Drabkin, A. S. (2014). Self-compassion and reactions to serious illness: The case of HIV. Journal of Health Psychology, 19(2), 218-229. doi:10.1177/1359105312467391

Brown, L., Bryant, C., Brown, V., Bei, B. e Judd, F. (2015). Selfcompassion, attitudes to ageing and indicators of health and wellbeing among midlife women. Aging \& Mental Health, 20(10) 1035-1043. doi:10.1080/13607863.2015.1060946

Burns, R. A., Anstey, K. J. e Windsor, T. D. (2011). Subjective well-being mediates the effects of resilience and mastery on depression and anxiety in a large community sample of young and middle-aged adults. Australian and New Zealand Journal of Psychiatry, 45(3), 240-248. doi:10.3109/00048674.2010.529604

Castilho, P. e Gouveia, J. P (2011). Auto-compaixão: Estudo da validação da versão portuguesa da escala da auto-compaixão e da sua relação com as experiências adversas na infância, a comparação social e a psicopatologia. Psychologica, 54, 203-230. Disponível em journals.uc.pt/psychologica/article/view/1106 http://impactum-

Clegg, A., Young, J., Iliffe, S., Rikkert, M. O. e Rockwood, K. (2013). Frailty in elderly people. The Lancet, 381(9868), 752-762. doi:10.1016/S0140-6736(12)62167-9

Diener, E. (1984). Subjective well-being. Psychological Bulletin, 95(3), 542-575.

Diener, E., Emmons, R. A., Larsen, R. J. e Griffin, S. (1985). The satisfaction with life scale. Journal of Personality Assessment, 49(1), 71-75. doi:10.1207/s15327752jpa4901_13

Del Duca, G. F., Silva, S. G., Thumé, E., Santos, I. S. e Hallal, P. C. (2012). Predictive factors for institutionalization of the elderly: A case-control study. Revista de Saúde Pública, 46(1), 147-153. doi:10.1590/S0034-89102012000100018

Falcão, D., Espírito Santo, H., Matreno, J., Fermino, S. e Guadalupe, S. (abril, 2012). Envelhecimento e funcionamento cognitivo: O papel da escolaridade e profissão. Artigo apresentado no I Congresso Internacional de Gerontologia Social dos Açores - Problemáticas e desafios: Construção duma nova realidade, Ilha Terceira, Açores. Disponível em http://repositorio.ismt.pt/handle/123456789/67

Freitas, S., Simões, M. R., Alves, L. e Santana, I. (2011). Montreal Cognitive Assessment (MoCA): Normative study for the Portuguese population. Journal of Clinical and Experimental Neuropsychology, 33(9), 989-996. doi:10.1080/13803395.2011.589374

Freitas, S., Simões, M. R., Martins, C., Vilar, M. e Santana, I. (2010). Estudos de adaptação do Montreal Cognitive Assessment (MOCA) para a população portuguesa. Avaliação Psicológica, 9(3), 345357.

Ferreira, P. L. (2000). Criação da versão portuguesa do MOS SF-36. Parte II - Testes de validação. Acta Médica Portuguesa, 13(3), 119-127.

Ferreira, P. L., Ferreira, L. N. e Pereira, L. N. (2012). Medidas sumário física e mental de estado de saúde para a população portuguesa. Revista Portuguesa de Saúde Pública, 30(2), 163-171. doi:10.1016/j.rpsp.2012.12.007

Galinha, I. C. e Pais-Ribeiro, J. L. (2005). Contribuição para o estudo da versão portuguesa da Positive and Negative Affect Schedule (PANAS): II - Estudo psicométrico. Análise Psicológica, 23(2), 219227. doi:10.14417/ap.84

Galinha, I. C., Pereira, C. R. e Esteves, F. (2014). Versão reduzida da escala portuguesa de afeto positivo e negativo - PANAS-VRP: Análise fatorial confirmatória e invariância temporal. Revista Psicologia, 28(1), 53-65. doi:10.17575/rpsicol.v28i1.622

Gilbert, P. (2005). Compassion and cruelty: A biopsychosocial approach. Em P. Gilbert (Ed.), Compassion: Conceptualisations, research and use in psychotherapy (pp. 9-74). London, UK: Routledge.

Gilbert, P. e Procter, S. (2006). Compassionate mind training for people with high shame and self-criticism: Overview and pilot study of a group therapy approach. Clinical Psychology and Psychotherapy, 13(6), 353-379. doi:10.1002/cpp.507

Guedea, M. T. D., Albuquerque, F. J. B., Tróccoli, B. T., Noriega, J. A. V., Seabra, M. A. B. e Guedea, R. L. D. (2006). Relação do bemestar subjetivo, estratégias de enfrentamento e apoio social em idosos. Psicologia: Reflexão e Crítica, 19(2), 301-308. doi:10.1590/S0102-79722006000200017 
Hall, C. W., Row, K. A., Wuensch, K. L. e Godley, K. R. (2013). The role of self-compassion in physical and psychological well-being. The Journal of Psychology: Interdisciplinary and Applied, 147(4), 311 323. doi:10.1080/00223980.2012.693138

HelpAge International. (2015). Global agewatch index 2015: Insight report [Online]. Acedido em 3, agosto, 2016, em http://www.helpage.org/global-agewatch/reports/globalagewatch-index-2015-insight-report-summary-and-methodology/

Carrilho, M. J. e Craveiro, M. L. (2015). A situação demográfica recente em Portugal. Em Instituto Nacional de Estatística (Ed.), Revista de Estudos Demográficos (Vol. 54, pp. 57-107).

Lawton, M. P. (1983). The varieties of wellbeing. Experimental Aging Research, 9(2), 65-72. doi:10.1080/03610738308258427

Lawton, M. P. (1989). Behavior-relevant ecological factors. Em K. W. Schaie e C. Schooler (Eds.), Social structure and aging: Psychological processes (pp. 57-78). Hillsdale, NJ: Lawrence Erlbaum Associates.

Kanning, M. e Schlicht, W. (2008). A bio-psycho-social model of successful aging as shown through the variable "physical activity." European Review of Aging and Physical Activity, 5(2), 79-87. doi:10.1007/s11556-008-0035-4

Kline, P. (1998). The new psychometrics: Science, psychology and measurement. New York, NY: Routledge.

Nasreddine, Z. S., Phillips, N. A., Bédirian, V., Charbonneau, S. Whitehead, V., Collin, I., ... Chertkow, H. (2005). The Montreal Cognitive Assessment, MoCA: A brief screening tool for mild cognitive impairment. Journal of the American Geriatrics Society, 53(4), 695-699. doi:10.1111/j.1532-5415.2005.53221.x

Neff, K. D. (2003a). Self-compassion: An alternative conceptualization of a healthy attitude toward oneself. Self and Identity, 2(2), 85-101. doi:10.1080/15298860309032

Neff, K. D. (2003b). The development and validation of a scale to measure self-compassion. Self and Identity, 2(3), 223-250. doi:10.1080/15298860309027

Neff, K. D. e Costigan, A. P. (2014). Self-compassion, wellbeing, and happiness. Psychologie in Österreich, 2(3), 114-119.

Neely, M. E., Schallert, D. L., Mohammed, S. S., Roberts, R. M. e Chen, Y.-J. (2009). Self-kindness when facing stress: The role of selfcompassion, goal regulation, and support in college students' wellbeing. Motivation and Emotion, 33(1), 88-97. doi:10.1007/s11031-008-9119-8

Organização Mundial da Saúde. (2015). Relatório mundial de envelhecimento e saúde [WHO reference number: WHO/FWC/ALC/15.01]. Disponível em http://www.who.int/iris/handle/10665/186468

Pavot, W. e Diener, E. (1993). Review of the satisfaction with life scale. Psychological Assessment, 5(2), 164-172. doi:10.1037/10403590.5.2.164

Pestana, M. H. e Gageiro, J. N. (2008). Análise de dados para ciências sociais: A complementaridade do SPSS (5. ${ }^{a}$ ed.). Lisboa: Edições Sílabo.
Phillips, W. J. e Ferguson, S. J. (2013). Self-compassion: A resource for positive aging. Journals of Gerontology, Series B: Psychological Sciences and Social Sciences, 68(4), 529-539. doi:10.1093/geronb/gbs091

Pinto, D. C. V. (2013). Por que vão os idosos para lares? Determinantes no internamento de pessoas maiores de 65 anos em instituições de longa permanência (Dissertação de mestrado, Universidade de Lisboa, Lisboa). Disponível em http://hdl.handle.net/10400.5/6622

Pinto-Gouveia, J., Duarte, C., Matos, M. e Fráguas, S. (2013). The protective role of self-compassion in relation to psychopathology symptoms and quality of life in chronic and in cancer patients. Clinical Psychology and Psychotherapy, 21(4), 311-323. doi:10.1002/cpp.1838

Ribeiro, O. e Paúl, M. C. (Coords.). (2011). Manual de envelhecimento activo. Lisboa: Lidel.

Sequeira, A. e Silva, M. N. (2002). O bem estar da pessoa idosa em meio rural. Análise Psicológica, 20(3), 505-516. doi:10.14417/ap.336

Simões, A. (1992). Ulterior validação de uma escala de satisfação com a vida (SWLS). Revista Portuguesa de Pedagogia, 26(3), 503-515.

Siqueira, M. M. M. e Padovam, V. A. R. (2008). Bases teóricas de bemestar subjetivo, bem-estar psicológico e bem-estar no trabalho. Psicologia: Teoria e Pesquisa, 24(2), 201-209. doi:10.1590/S0102 37722008000200010

Teixeira, L. M. F. (2010). Solidão, depressão e qualidade de vida em idosos: Um estudo avaliativo exploratório e implementação-piloto de um programa de intervenção (Dissertação de mestrado, Universidade de Lisboa, Lisboa). Disponível em http://hdl.handle.net/10451/2608

Terry, M. L. e Leary, M. R. (2011). Self-compassion, self-regulation, and health. Self and Identity, 10(3), 352-362. doi:10.1080/15298868.2011.558404

Vitorino, L. M., Paskulin, L. M. G. e Vianna, L. A. C. (2013). Quality of life of seniors living in the community and in long term care facilities: A comparative study. Revista Latino-Americana de Enfermagem, 21(Esp.), 3-11. doi:10.1590/S010411692013000700002

Ware, J. E. e Sherbourne, C. D. (1992). The MOS 36-Item Short-Form Health Survey (SF-36): I. Conceptual framework and item selection. Medical Care, 30(6), 473-483. doi:10.2307/3765916

Watson, D., Clark, L. A. e Tellegen, A. (1988). Development and validation of brief measures of positive and negative affect: The PANAS scales. Journal of Personality and Social Psychology, 54(6), 1063-1070. doi:10.1037/0022-3514.54.6.1063

World Health Organization. (2002). Active ageing: A policy framework. [WHO reference number: WHO/NMH/NPH/02.8]. Disponível em http://www.who.int/iris/handle/10665/67215

Yarnell, L. M., Stafford, R. E., Neff, K. D., Reilly, E. D., Knox, M. C. e Mullarkey, M. (2015). Meta-analysis of gender differences in selfcompassion. Self and Identity, 14(5), 499-520. doi:10.1080/15298868.2015.1029966 